\title{
Prenols and Ubiquinones in Single-strain and Mated Cultures of Blakeslea trispora
}

\author{
By J. D. BU'LOCK AND A. U. OSAGIE \\ Microbial Chemistry Laboratory, Department of Chemistry, \\ The University, Manchester, MI3 9PL
}

(Received 22 August 1972; revised 27 November 1972)

\section{SUMMARY}

The prenols of Blakeslea trispora were found to be a mixture of $\mathrm{C}_{60}-\mathrm{C}_{90}$ prenols and $\alpha$-dihydroprenols with $\mathrm{C}_{70}$ as the main type; the principal ubiquinones were ubiquinone-9 and its (side-chain) dihydro-derivative. A minus strain contained higher levels of prenols, ubiquinones, ergosterol, and $\beta$-carotene than a plus strain, and the levels of all four types of isoprenoid were higher again in mated cultures. The overall increase in isoprenoid synthesis in mated cultures was sometimes tenfold but the different isoprenoid types were not all equally affected by the mating process; possible roles for the different types in sexual differentiation are considered.

\section{INTRODUCTION}

The accumulation of carotenoids in the differentiated sexual structures of many mucoraceous fungi (Burnett, 1965) and in submerged agitated cultures of opposite mating types of heterothallic species, such as Blakeslea trispora, is due to the action of the trisporic acids which oppositely sexed strains collaboratively produce. In particular, Thomas, Harris, Kirk $\&$ Goodwin (I967) showed that the enhanced synthesis of both $\beta$-carotene and ergosterol caused by additions of trisporic acids to single strains of $B$. trispora is blocked if protein synthesis has been prevented by prior addition of cycloheximide and, because this inducerlike action of the trisporic acids affects both carotenoid and steroid synthesis, they concluded that the induction primarily affected an enzyme or enzymes early in the isoprenoid biosynthetic pathway.

Since the trisporic acids are responsible for eliciting all the early stages of sexual differentiation in the Mucorales (van den Ende, I968; Austin, Bu'Lock \& Gooday, I969) a wider understanding of their biochemical action is desirable, and the other branches of the isoprenoid biosynthetic pathway offer an obvious starting point. In this paper we describe the characterization of the functionally important ubiquinones (UQ- $n$, where $n$ indicates the number of isoprene residues in the side-chain, e.g. UQ-9) and prenols (polyprenol alcohol, PP- $n$, where $n$ indicates the number of isoprene residues, lettered $\alpha, \ldots, \psi, \omega$ as in $\alpha-\mathrm{H}_{2} \mathrm{PP}-\mathrm{I} 5$, otherwise, 2,3-dihydropentadecaprenol). Their separation permits measurements of the four main types of isoprenoid in Blakeslea trispora ( $\beta$-carotene, ergosterol, UQ and PP) and comparisons of their levels in single-strain, plus or minus, and mated (plus with minus) cultures.

\section{METHODS}

General. The strains of Blakeslea trispora used were NRRL2895 (plus) and 2896 (minus), and the culture procedures are fully described elsewhere (Bu'Lock \& Winstanley, I97I). For the present work, shake cultures were grown on $10 \%$ commercial malt extract for 
7 days after inoculation. Throughout, ether means diethyl ether, petrol means light petroleum b.p. 40 to $60{ }^{\circ} \mathrm{C}$, solvent ratios are $\mathrm{v} / \mathrm{v}$ and $\%$ is $\mathrm{w} / \mathrm{v}$.

Characterization of isoprenoids. Because the most exacting task was characterization of the PP fraction, which can occur both free and esterified, the well-established saponification work-up (Burgos, Hemming, Pennock \& Morton, I963; Stone, Butterworth \& Hemming, 1967) was adopted for this aspect of the work even though this gives low recoveries of $\beta$-carotene, ergosterol and UQ. The non-saponifiable product was taken up in petrol, and the sterols mostly precipitated by refrigeration, before subjecting the mixture to chromatography on acid-washed alumina (grade III), first in 3:97 ether:petrol to elute carotenoids, etc., and then in 8:92 ether: petrol to elute a fraction containing PP and UQ. Direct further purification of the PP at this stage was impeded by the presence of ubichromenols. This fraction was therefore acetylated with pyridine-acetic anhydride (overnight) and rechromatographed; I:99 ether:petrol elution gave a clean PP-acetate fraction and 10:90 ether:petrol gave a UQ fraction. Each fraction was finally purified by thin-layer chromatography (t.l.c.) (Merck silica gel F 254 eluting with dichloromethane). The PP-acetate fraction was alternatively reconverted to free PP by saponification before final t.l.c.

Visualization on thin-layer chromatograms was with iodine, phosphomolybdic acid (10\% in ethanol), anisaldehyde $\left(5 \%\right.$ with $5 \% \mathrm{H}_{2} \mathrm{SO}_{4}$ in ethanol: $\beta$-carotene, blue-green; ergosterol, red-brown; UQ, deep green; PP, deep green) or reduced methylene blue (reoxidized by UQ). For comparisons with authentic materials the most useful thin-layer system was the two-dimensional method of Dunphy et al. (1967), which resolved the various categories very adequately but did not tend to separate different homologues.

Mass spectra were determined on the AEI MS-I 2 instrument operating at $250^{\circ} \mathrm{C}$ and 6 or $8 \mathrm{kV}$; n.m.r. spectra were obtained on the Varian HA-I 00 in deuterochloroform with tetramethylsilane as internal reference. Authentic UQ-9 and a dolichol $\left(\alpha-\mathrm{H}_{2} \mathrm{PP}\right)$ were kindly provided by Dr F. W. Hemming.

Estimation of isoprenoids. Assays of $\beta$-carotene, ergosterol, and UQ were carried out on mycelium from single shake flasks, which was filtered off, pressed dry on paper, ground to a powder under liquid $\mathrm{N}_{2}$, and stirred for $30 \mathrm{~min}$ with $150 \mathrm{ml}$ of acetone. The acetone slurry was filtered, the residue being used for dry-weight measurement, and the filtrate evaporated under reduced pressure to give an extract which was taken up in ether (I00 $\mathrm{ml})$ for spectrophotometric assay of $\beta$-carotene $\left(E_{1 \mathrm{~cm}}^{1 \%} 2500\right.$ at $\left.45^{2} \mathrm{~nm}\right)$ and ergosterol $\left(E_{1 \mathrm{~cm}}^{10 \%} 340\right.$ at $280 \mathrm{~nm}$ ). The ether extract was then evaporated and the residue taken up in petrol for chromatography as above, eluting first with $3: 97$ and then with 8:92 ether:petrol. The latter fraction was evaporated and taken up in ethanol; the UQ content was determined from the change in extinction at $275 \mathrm{~nm}$ after addition of a little solid sodium borohydride to the cuvette (for UQ-9, $\Delta E_{1 \mathrm{~cm}}^{1 \%}$ (ox.-red.) I 56 at $275 \mathrm{~nm}$ ). Assays of up to five replicates at a time gave the variance indicated in Table 2.

Assays of PP were carried out by means of the saponification procedure with mycelium from at least five flasks. The 8:92 ether:petrol fraction from the non-saponifiable material was acetylated and subjected to t.l.c. on washed Merck F-254 silica gel analytical plates, applied as a $\mathrm{I} 6 \mathrm{~cm}$ band and eluting with dichloromethane; the PP-acetate zone $\left(R_{F} 0.50\right)$ was scraped off and eluted and the PP-acetate was estimated by direct weighing. Usually about 0.5 to $3.5 \mathrm{mg}$ of PP-acetate was weighed, and the corresponding mycelial dry wt was obtained directly from replicate flasks; the PP-acetate obtained by this procedure on a somewhat larger scale was over $90 \%$ pure by n.m.r. observations and loss in recycling tests was under $20 \%$. The main source of variability in the assays was apparently in the intensity of the mating response, and could most easily be judged by the $\beta$-carotene assays which 
were very dependent upon the history and condition of the two inocula. The results in Table 2 are typical of those obtained when the response was high, but even with less good responses the qualitative picture was consistent throughout.

\section{RESULTS}

Identity of the ubiquinones. The UQ fraction behaved identically with authentic material in chromatography, colour reactions, and u.v. spectrometry. In the mass spectrum the strongest molecular ion group was at $m+/ e 794$ and 796 (with attendant isotope peaks) corresponding to ubiquinones with 9 isoprene units, with a series of low-intensity fragmentation clusters (M-69- $n \times 68)$ and a prominent fragment at $m+/ e 235$ (Muraca et al. 1967). In the molecular ion group, $m+/ e 794$ corresponds to UQ-9. The peak at $m+/ e 796$ could be due to ubiquinol formed in the instrument or to a ubiquinone with a single reduced isoprene residue in the side-chain, UQ- $-9\left(\mathrm{H}_{2}\right)$. A sample dissolved in ether was shaken vigorously with aqueous sodium dithionite under nitrogen, to convert quinones into quinols, and the ether layer evaporated under nitrogen and submitted to mass spectrometry. The molecular ion group now appeared at $m+/ e 796$ and 798 , showing that the original material was a mixture of UQ-9 and UQ- $9\left(\mathrm{H}_{2}\right)$.

During the work-up a ubichromenol fraction was encountered but there was no evidence that this was not an artefact.

Ubiquinones with side-chains shorter than that of the UQ-9, UQ-9 $\left(\mathrm{H}_{2}\right)$ mixture will have molecular ions coinciding with the $+\mathrm{I}$ isotope peaks of the fragmentation series of the larger molecules; detailed examination of the peak clusters with this principle in mind suggested that shorter $U Q$ in our material were very minor components. More unambiguously, UQ-Io, UQ- $\mathrm{IO}\left(\mathrm{H}_{2}\right)(\mathrm{M}$ at $m+/ e 862,864)$ was present in less than $\mathrm{I} / 50$ of the amount of UQ-9, UQ-9 $\left(\mathrm{H}_{2}\right)$.

Identity of the prenols. The PP fraction was authenticated by chromatography, colour reactions, and i.r., n.m.r., and mass spectra. It was a mixture in approximately 3:5 ratio, of true polyisoprenyl alcohols $\mathrm{H} .\left[\mathrm{C}_{5} \mathrm{H}_{8}\right]_{n} . \mathrm{OH}$ and corresponding dihydrocompounds, each type comprising a range of chain lengths. In the mass spectrum the molecular ions, the fragmentation peaks, and the associated isotope peaks, formed quite complex clusters. For example, for the (predominant) species with $n=14$ the molecular ion cluster comprised

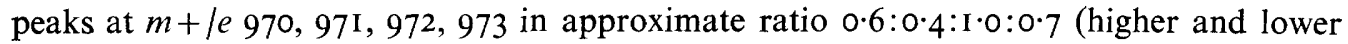
neighbours $<0 \cdot 2$ ). The $\mathrm{H}_{2} \mathrm{PP}$ behaved as expected if the proximal (HO-terminal, $\alpha$ ) isoprene residue was saturated, and fragmented mainly by losses of $C_{5}$ units from the distal $(\omega)$ end giving a series $(\mathrm{M}-69-n \times 68)$; hence the $+\mathrm{I}$ isotope peaks of this series coincided with the molecular ions of shorter $\mathrm{H}_{2} \mathrm{PP}$ and the fragmentation peaks themselves coincided with the $+\mathrm{I}$ isotope peaks of shorter chain PP. In contrast the true PP fragmented mainly by initial loss of $\mathrm{H}_{2} \mathrm{O}$ giving a series (M-I 8- $\left.n \times 68\right)$; this difference was even more marked in the mass spectra of the acetates. With these observations as a guide (Stone et al. 1967; Gough \& Hemming, 1970) and noting that the relative intensities of the peaks did not change significantly in spectra run serially (probe temperature $250^{\circ} \mathrm{C}$ ), the approximate chain-length composition of the $\mathrm{PP}-\mathrm{H}_{2} \mathrm{PP}$ mixture was worked out as in Table $\mathrm{I}$; the main componeri: was $\mathrm{H}_{2} \mathrm{PP}-\mathrm{I} 4$.

The i.r. spectrum of the mixture agreed with expectation for a predominance of $\alpha \mathrm{H}_{2} \mathrm{PP}$ with mainly $c i s$-double bonds ( $v_{\max } 886$, I060, I090, I I 30, I $\left.240 \mathrm{~cm}^{-1}\right)$. More critically, both the prenol mixture and the derived acetates gave well-resolved n.m.r. spectra (at $100 \mathrm{~Hz}$ ) which agreed with expectations (Feeney \& Hemming, 1967) and in particular showed that 
Table I. Chain-length composition of the 3:5 PP- $\alpha \mathrm{H}_{2} P P$ mixture from Blakeslea trispora

$\begin{array}{lccccrrc}\text { No. of isoprene units... } & 12 & 13 & 14 & 15 & 16 & 17 & 18 \\ \text { Approximate } \% & \text { Trace } & 15 & 70 & 10 & 3 & 1 & \text { Trace }\end{array}$

Table 2. Isoprenoid levels $(\mathrm{mg} / \mathrm{g})$ in Blakeslea trispora

\begin{tabular}{lcccc}
\multicolumn{1}{c}{ Strain } & Ergosterol* & $\beta$-Carotene* & UQ* & PP $\dagger$ \\
plus & $3.5 \pm 0.3$ & $0.17 \pm 0.03$ & $0.09 \pm 0.03$ & 0.06 \\
minus & $9 \cdot 2 \pm 0.9$ & $0.8 \pm 0.1 \mathrm{I}$ & $0.26 \pm 0.03$ & 0.10 \\
plus with minus & $5 \mathrm{I} \cdot 0 \pm 8$ & $\mathrm{II} \cdot 0 \pm \mathrm{I} \cdot 7 \ddagger$ & $0.52 \pm 0 . \mathrm{II}$ & 0.35 \\
Average increase & $\times 8$ & $\times \mathbf{2 2}$ & $\times 3$ & $\times 4$
\end{tabular}

* Mean and S.D. of five samples each of $\mathrm{I}$ to $\mathrm{I} \cdot 5 \mathrm{~g}$ dry weight.

$\dagger$ From saponification of 10 to $12 \mathrm{~g}$ dry weight.

$\ddagger$ Also approx. $20 \mathrm{mg} / \mathrm{g}$ trisporic acids $\equiv 35 \mathrm{mg} \beta$-carotene.

(i) in the mixture it was the $\alpha$-terminal isoprene residue that was predominantly saturated (for the acetate, $\mathrm{CH}_{3}$-(sat) doublet at $\tau$ 9.07, 9.15; (sat)- $\mathrm{CH}_{2}$ (sat) multiplet at $\tau$ 8.75; AcO- $\mathrm{CH}_{2}$ (sat) triplet at $\tau 6.30 ; \mathrm{AcO}-\mathrm{CH}_{2}$ (allylic) doublet at $\tau 5.90$; relative integrals in approximate agreement with the 3:5 mixture deduced from the mass spectra); (ii) the methyl signals at $\tau 8.34$ and $8.4 \mathrm{I}$, due to methyl on cis- and trans-double bonds respectively (one of each kind on the $\omega$-residue) had integrated intensities in the ratio 9.5:4.5; allowing for incomplete resolution of the smaller peak from the larger, for the presence of some $\alpha$ unsaturated residues, and for the distribution of chain lengths (cf. Table I) this corresponded to three internal trans-residues (theoretical for this case if fully resolved, 10:4; for two internal trans, II:3).

Isoprenoid synthesis in single strains and mated cultures. Analytical data for plus and minus strains and for mated cultures of Blakeslea trispora are given in Table 2. All types of isoprenoid occurred at higher levels in our minus strain than in the plus, and all were further increased in the mated cultures. Proportionately the greatest increase was in the $\beta$-carotene but in terms of quantity the effect on ergosterol was largest. Because the growth rates of the plus and minus strains were not very different when they were grown separately, it is reasonable to assume that the two were present in very approximately equal proportions in the mated cultures, and hence to compare results for the latter with the averages for the two separate strains. With a good mating response such as Table 2 exemplifies, the overall increase in isoprenoid biosynthesis was about tenfold, but this was not equally reflected in the four separate categories.

\section{DISCUSSION}

The side-chains of ubiquinones are formed by the repeated trans-coupling of isoprene residues; in some ubiquinones one of the side-chain units is reduced. Table 3 summarizes data from Law, Threlfall \& Whistance (197I) on the predominant UQ types in various fungi. The UQ-6 of Saccharomyces cerevisiae seems to be exceptional, but the predominance of UQ-9 or UQ-I0, with or without side-chain reduction, does not seem to have any clearcut taxonomic basis, at least on the present restricted range of data. The closest relative to Blakeslea trispora studied by these workers was Phycomyces blakesleeanus, in which UQ-9 predominates; the difference in $B$. trispora is the presence of an approximately equal amount of UQ-9 $\left(\mathrm{H}_{2}\right)$.

Comparable data for fungal prenols are even more limited (Table 4). These differ from 
Table 3. Fungal ubiquinones (from Law et al. I97I)

Dominant UQ

UQ-IO

UQ-IO $\left(\mathrm{H}_{2}\right)$

UQ-9

$\mathrm{UQ}-9\left(\mathrm{H}_{2}\right)$

UQ-6

Genera

Aspergillus

Alternaria, Aspergillus, Claviceps, Neurospora

Agaricus, Aspergillus, Penicillium, Phycomyces, Rhodotorula

Penicillium

Saccharomyces

Table 4. Fungal prenols

\begin{tabular}{|c|c|c|c|c|c|c|}
\hline \multirow[b]{2}{*}{ Source } & \multicolumn{2}{|c|}{ Total $\mathrm{C}_{5}$ units } & \multirow{2}{*}{$\begin{array}{l}\text { Biosynthetically } \\
\text { trans- } C_{5} \text {-units }\end{array}$} & \multirow{2}{*}{$\begin{array}{l}\text { Hydrogenated } \\
\text { residues }\end{array}$} & \multirow{2}{*}{$\begin{array}{c}\text { Other } \\
\text { modifications }\end{array}$} & \multirow[b]{2}{*}{ References } \\
\hline & Predominant & Range & & & & \\
\hline $\begin{array}{l}\text { Saccharomyces } \\
\text { cerevisiae }\end{array}$ & $?$ & I3-18 & $3 ?$ & $\alpha$ & 一 & Dunphy et al. 1967 \\
\hline $\begin{array}{l}\text { Aspergillus } \\
\text { fumigatus }\end{array}$ & $20-22$ & I 8-24 & 4 & $\alpha \psi \omega$ & - & $\begin{array}{l}\text { Stone \& Hemming, } \\
1967\end{array}$ \\
\hline A. niger & $20,2 \mathrm{I}$ & I 8-24 & 4 & $\alpha \psi$ & $\begin{array}{l}\omega \text { is methylene- } \\
\text { dihydro }\end{array}$ & $\begin{array}{l}\text { Barr \& Hemming, } \\
\text { I972 } a\end{array}$ \\
\hline $\begin{array}{c}\text { Blakeslea } \\
\text { trispora }\end{array}$ & 14 & I $2-18$ & 4 & None and $\alpha$ & - & Present work \\
\hline
\end{tabular}

the UQ side-chains in arising by cis-coupling steps from precursors with either three or four trans-coupled residues. The prenols of Blakeslea trispora have three internal trans residues, i.e. biogenetically four trans units. The true PP component of $B$. trispora represents a type hitherto found only in angiosperms (castaprenols) while the $\alpha-\mathrm{H}_{2} \mathrm{PP}$ type is more normal; however, the $\alpha-\mathrm{H}_{2} \mathrm{PP}$ of yeast is said to have only two internal trans residues, like the mammalian dolichols. The Aspergillus prenols have the same biogenetic stereochemistry as those of $\boldsymbol{B}$. trispora but they are also reduced, and in one case alkylated, at the distal residues. Bacterial prenols are different again, with shorter chain lengths and only two internal trans residues; occasionally the $\alpha$-residue is saturated but this is atypical (Scher, Lennarz \& Sweeley, I968; Durr \& Habbal, 1972).

The branching of pathways of isoprenoid metabolism leading to the compounds studied here is schematized in Fig. I. All the reactions are intensified in the mated cultures, in keeping with the views of Thomas et al. (1967) on the location of the inducer action of trisporic acids, but to different degrees (Table 2), which must reflect the capacity of later enzymes in the different branches of the sequence to handle an overall increase in substrate levels. The possible roles of each sequence branch in the mating process, and in particular in those parts of the process which can be induced directly in single strains by added trisporic acids, merit some consideration.

Exogenous steroids are essential for reproductive development in Oomycetes and endogenous sterols may be similarly implicated in a wider range of fungi (Hendrix, 1965; Nelson, Huisingh \& Webster, I967; Elliott, I969); in Achlya specific sterols have a hormone function (Barksdale, 1969). More generally, a steroid function in the lipids of membrane structures controlling permeability may be anticipated and could well have a role in the post-contact phases of the sexual process in Mucorales.

The carotenoid pathway is critical for sexual development in these fungi because $\beta$ carotene is itself the precursor from which the trisporic acids are formed when plus and minus strains collaborate (Austin, Bu'Lock \& Drake, 1970). However, an actual accumulation of $\beta$-carotene in mated cultures is not required for such a role, and though such accumulations are conspicuous in many Mucorales, and particularly in Blakeslea trispora 


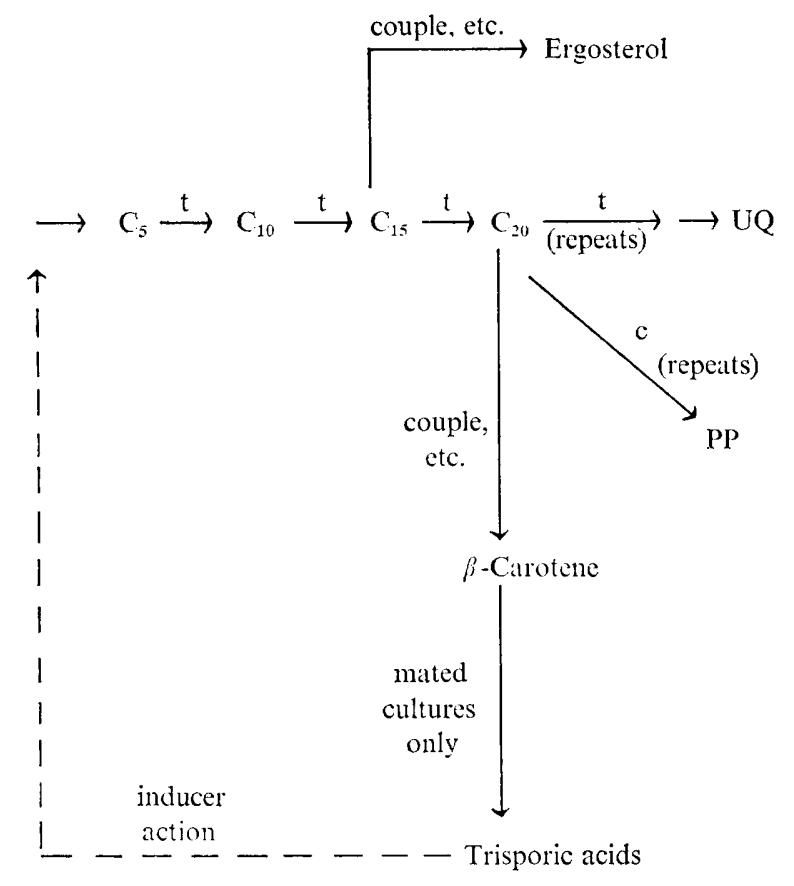

Fig. I. Schematized isoprenoid synthesis in Blakeslea trispora. $\mathrm{t}$, trans-mechanism; c, cis-mechanism.

(Ciegler, 1965), other species do not accumulate carotenoids visibly (Burnett, 1965), though the converse, i.e. the strict absence of carotenoids in such species, has not been demonstrated and seems a priori unlikely. Carotenoids may have a direct function in the later stages of the mating process since they have been suggested as precursors of the zygospore coat-substance (G. W. Gooday, personal communication).

The general functions of UQ in respiration and of PP as carriers for the synthesis of extramembrane polymers (e.g. in Aspergillus; Barr \& Hemming, 1972 $b$ ) are well established, and it is noteworthy that the synthesis of both materials in Blakeslea trispora seems to be more tightly regulated, and less drastically affected by the mating effect, than the syntheses of $\beta$-carotene and ergosterol. However, zygophore development in Mucorales involves, as may readily be observed in plate cultures, the capacity for rather rapid outgrowths of a specialized hyphal type, with specific surface properties, which branch out from mycelia at points rather remote from the hyphal tips where wall synthesis is normally concentrated. In such circumstances an additional supply of UQ and PP could well be directly functional in zygophore outgrowth.

To these direct consequences of the trisporic-acid-mediated induction in the isoprenoid pathway we must add the indirect effects ensuing after a five- to tenfold increase in the consumption of acetyl-CoA, ATP and NADPH in this pathway, which will obviously be extensive. However, it remains for further investigation to establish whether the accumulated biochemical effects of a single-point inducer action are wholly sufficient to account for the range of phenomena elicited by trisporic acids in the mating process. 


\section{REFERENCES}

Austin, D. J., Bu'Lock, J. D. \& Gooday, G. W. (1969). Trisporic acids: sexual hormones from $M u c o r$ mucedo and Blakeslea trispora. Nature, London 223, $1178-1179$.

Austin, D. J., Bu'Lock, J. D. \& Drake, D. (1970). The biosynthesis of trisporic acids from $\beta$-carotene via retinal and trisporol. Experientia 26, 348-349.

Barksdale, A. W. (1969). Sexual hormones of Achlya and other fungi. Science, New York 166, 83I-837.

Barr, R. M. \& Hemming, F. W. (I972a). Polyprenols of Aspergillus niger. Biochemical Journal 126, I193I 202.

Barr, R. M. \& Hemming, F. W. (I972 b). Polyprenol phosphate as an acceptor of mannose from guanosine diphosphate mannose in Aspergillus niger. Biochemical Journal 126, 1 203-1 208.

Bu'Lock, J. D. \& Winstanley, D. J. (1971). Trisporic acid production by Blakeslea trispora and its promotion by barbiturate. Journal of General Microbiology 69, 39I-394.

Burgos, J., Hemming, F. W., Pennock, J. F. \& Morton, R. A. (1963). Dolichol: a naturally-occurring $C_{100}$ isoprenoid alcohol. Biochemical Journal 88, 470-482.

BuRNETT, J. H. (1965). Functions of carotenoids other than in photosynthesis. In Chemistry and Biochemistry of Plant Pigments, pp. 38I-403. Edited by T. W. Goodwin. London: Academic Press.

Ciegler, A. (1965). Microbial carotenogenesis. Advances in Applied Microbiology 7, I-34.

Dunphy, P. H., Kerr, J. D., Pennock, J. F., Whittle, K. J. \& Feeney, J. (1967). The plurality of long-chain isoprenoid alcohols from natural sources. Biochimica et biophysica acta 136, I36-147.

DurR, I. F. \& HabBal, M.Z. (1972). The biosynthesis of $\mathrm{C}_{55}$ polyprenols by a cell-free preparation of Lactobacillus plantarum. Biochemical Journal 127, 345-359.

Elliott, C. G. (1969). Effects of inhibitors of sterol synthesis on growth of Sordaria and Phytophthora. Journal of General Microbiology 56, 33 I-343.

VAN DEN ENDE, H. (1968). Relationship between sexuality and carotene synthesis in Blakeslea trispora. Journal of Bacteriology 96, I 298-1 303.

Feeney, J. \& Hemming, F. W. (1967). Nuclear magnetic resonance spectrometry of naturally occurring polyprenols. Analytical Biochemistry 20, I-I 5 .

Gough, D. P. \& Hemming, F. W. (1970). The characterization and stereochemistry of biosynthesis of dolichols in rat liver. Biochemical Journal I18, I63-I 66.

Hendrix, J. W. (1965). Influence of sterols on growth and reproduction of Pythium and Phytophthora. Phytopathology 55, 790-797.

Law, A., Threlfall, D. R. \& Whistance, G. R. (I97I). Isoprenoid phenol and quinone precursors of ubiquinones and dihydroubiquinones in fungi. Biochemical Journal 123, 33I-339.

Muraca, R. F., Whittick, J. S., Daves, G. D., Frils, P. \& Folkers, K. (I967). Mass spectra of ubiquinones and ubiquinols. Journal of the American Chemical Society 89, I 505-1508.

Nelson, R. R., Huisingh, D. \& Webster, R. K. (1967). Sexual differentiation in Cochliobolus carbonum as influenced by inhibition and repair of steroid biosynthesis. Phytopathology 57, 1081-1085.

Scher, M., Lennarz, W. J. \& Sweeley, C. C. (I968). The biosynthesis of mannosyl-I-phosphorylpolyisoprenol in Micrococcus lysodeikticus. Proceedings of the National Academy of Sciences of the United States of America 59, I313-I 320.

Stone, K. J., Butterworth, P. H. W. \& Hemming, F. W. (1967). Characterization of the hexahydropolyprenols of Aspergillus fumigatus. Biochemical Journal ro2, 443-445.

Stone, K. J. \& Hemming, F. W. (I967). The stereochemistry of hexahydroprenol, ubiquinone and ergosterol biosynthesis in the mycelium of Aspergillus fumigatus. Biochemical Journal 104, 43-56.

Thomas, D. M., Harris, R. C., KIRK, J. T. O. \& Goodwin, T. W. (1967). The mode of action of trisporic acid. Phytochemistry 6, 36I-366. 